\title{
Recent Advances of Probiotics in Food Industry
}

\author{
Shahrukh Husain, Akshita Gupta, Hitesh Shetty, Kritika Sehgal, Smriti Gaur * \\ Department of Biotechnology, Jaypee Institute of Information Technology, A-10, Sector-62, Noida -201307, Uttar Pradesh, India
}

Address for Correspondance: Smriti Gaur, smriti.gaur@jiiit.ac.in

\section{Keywords}

Probiotics; LAB; GRAS; Prebiotics.

\begin{abstract}
Probiotics has a long history not only in the health but also in food industry. It started when Metchnikoff for the first time noticed the reduction of toxin producing bacteria in the gut through the intake of yogurt which contains Lactobacilli. Later, Tissier observed that gut microbiota from healthy breast fed infants were dominated by rods with bifid shaped bacteria, which were absent when infant is suffering from diarrhea. After many years of research it was found that probiotics have many health benefits over various other food supplements like better nutrient digestion, anti carcinogenic activity, antioxidant activity, reduce serum cholesterol and more. These probiotics are GRAS recognized with no pathogenic or virulent properties towards the host. Majorly, Probiotics are divided in two types LAB and bifid bacterium species. But some yeast are also a part of probiotics, like Saccharomyces boulardii and Saccharomyces cerevisiae. These yeasts play a major role in food industry like in the making of fermented food products. Also they have a symbiotic relationship with probiotic bacteria which enhances their functioning. Probiotics dominate in dairy industry like in cheese making, yogurt making and improving properties of milk for lactose intolerant person. Other non dairy based products include meat, juices, cereals, and vegetables. Recent advances are taking place in the food industry to incorporate probiotics for the betterment of health in a more natural way. Like introduction of prebiotics (aloe vera, fruit salad and paneer spread) for more convenience in transferring the probiotics in the human body but also to increase their activity. (c) 2016 iGlobal Research and Publishing Foundation. All rights reserved.
\end{abstract}

Conference Proceedings: International Conference on Advances in Plant and Microbial Biotechnology (PMB2017); JIIT, Noida: February 02-04, 2017

Indo Global Journal of Pharmaceutical Sciences( ISSN 22491023 ; CODEN- IGJPAI; NLM ID: 101610675) indexed and abstracted in EMBASE(Elsevier), SCIRUS(Elsevier),CABI, CAB Abstracts, Chemical Abstract Services(CAS), American Chemical Society(ACS), Index Copernicus, EBSCO, DOAJ, Google Scholar and many more. For further details, visit http://iglobaljournal.com 